\title{
The Influence of Environment on the Hydrous Ferric Oxide Composite Resin Adsorption of $\beta$-Naphthalene Sulfonic Acid
}

\author{
Fenggang $\mathrm{LI}^{1, \mathrm{a}}$, Peng $\mathrm{YAN}^{1, \mathrm{~b}}$, Caixia $\mathrm{JU}^{1, \mathrm{c}}$, Ningning $\mathrm{LI}^{1, \mathrm{~d}}$, Yanan $\mathrm{LI}^{1, \mathrm{e}}, \mathrm{Yu}$ \\ ZHANG $^{1, f}$, Fengbin ZHENG $^{1, g}$, Changhai $\mathrm{LI}^{2, \mathrm{~h}}$ \\ ${ }^{1}$ School of Chemistry and Chemical engineering, Zhaozhuang University, Zhaozhuang 277160, \\ China \\ ${ }^{2}$ Department of Chemistry and Chemical engineering, Binzhou University, Binzhou 256603, China \\ alfgang2005@163.com, byanpenglzu@163.com, cjucaixia2004@163.com, \\ d794231475@qq.com,,1468432570@qq.com, ${ }^{\mathrm{f}}$ 123269@163.com, ${ }^{\mathrm{h}}$ lichanghai2000@163.com
}

Keywords: Hydrated Ferric Oxide; pH; Exchange Resin; Adsorption; $\beta$-naphthalene Sulfonic Acid Abstract. A new type of macroporous weakly basic anion exchange resin (HFO D301R) was used to adsorption of naphthalene sulfonic acid in different environments. The effects of temperature, salt concentration and $\mathrm{pH}$ value of the solution on HFO- naphthalene sulfonic acid resin adsorption effect were studied The results show that HFO-D301R maximum adsorption capacity of $\beta$-naphthalene sulfonic acid can reach $235 \mathrm{mg} / \mathrm{g}$ at $10{ }^{\circ} \mathrm{C}$. The increase of salt solution concentration (sodium chloride or sodium sulfate) restrain adsorption of iron composite resin on $\beta$ - naphthalene sulfonic acid; The $\mathrm{pH}$ value of the solution also affect the adsorption capacity of the iron composite resin, and the best result of adsorption was obtained at $\mathrm{pH}=4$.

\section{Introduction}

$\beta$-naphthalene sulfonic acid sodium salt ( $\beta$-salt) producted as dye intermediates results in a large number of $\beta$ - salt mother liquor. The color of mother liquor is deep because it contains $3 \%$ of the $\beta$-naphthalene sulfonic acid , 3\% of the sulfuric acid and a small amount of Sulphur trioxide. The $\mathrm{pH}$ of mother liquor is lower than 3. Today, the main processing technologies have chemical oxidation, membrane separation and extraction[1].These presence methods have many shortcomings, which include high consumption of oxidizing agent; easily polluting the membrane materials whose life is low; high consumption of extraction agent; difficult reextraction process currently, the harmlessness of the $\beta$-naphthalene sulfonic acid wastewater cannot effectively realize. At present, the ion exchange resin method which are separated of organic and inorganic matter in aqueous solution have some rarely successful examples $[2,3]$. However, there is not research report that $\beta$ - naphthalene sulfonic acid/sulfuric acid/sulfite mixture is adsorpted by weakly basic anion exchange resin composited on hydrated ferric oxide. In this article, the main object of study is macroporous weakly basic anion exchange resin (domestic, D301) as the carrier to compound a kind of new adsorption material according to the literature[4]. which is evaluated by ferric oxide composite resin exchange absorption of naphthalene sulfonic acid in different surroundings : the temperature, $\mathrm{pH}$, salt, etc.

\section{Experimental Section}

\section{Reagent and Instrument}

$\beta$-naphthalene sulfonic acid (provided by the zaozhuang terry fine chemical Ltd.), D301R macroporous styrene type weak basic anion exchange resin (Nankai university chemical plant), Deionized water (homemade), Acetonitrile (chromatography), methanol (chromatography) and analytic reagent: $\beta$-naphthalene sulphenic sodium, potassium permanganate.ferrous sulfate, acetone,sodium hydroxide,sodium chloride,sodium sulfate;Low temperature constant temperature water bath oscillator SHA-1 (frozen) ( jintan science and analysis instruments Co. Ltd), High performance liquid chromatography (HPLC) waters2695、PHS-3B pH meter (Shanghai Mathematical Sciences instruments Co. Ltd), ultrasonic degassing machine SK250H (Shanghai Kedao Ultrasonic Instrument Co. Ltd), Suction filter device AL - 01P(Beijing Liyuan electronic 
instrument Technology and Trade Co. Ltd), electronic analytical balance ( made by Metter-Toledo Group)

\section{Chromatographic condition}

Chromatographic column: the inner diameter $3.9 \mathrm{~mm}, 150 \mathrm{~mm}$ long stainless steel tube, symmetryshieldTM RP18, $5 \mu \mathrm{m}$, Column temperature $35^{\circ} \mathrm{C}$, mobile phase: water:acetonitrile mixture (7:3); detection wavelength:275 nm;Flow rate: $0.8 \mathrm{~mL} / \mathrm{min}$. Workstation: Empower2.4; Injection Volume: 10 $\mu$; Detector: Water2996 Phtoiode Array Detector.

\section{The hydrated ferric oxide resin synthesis}

A certain amount of the pretreated resin carrier (30g of macroporous weakly basic anion exchange resin D301R) is made composite resin according to the literature [4]. composite resin is abbreviated HFO - D301R.

\section{Static absorption experiment and Calculation of adsorption quantity}

A certain amount of different concentrations of $\beta$-naphthalene sulfonic acid is added into the iodine flask, this time a certain amount of composite resin is added. Then it will be oscillated at the selected constant temperature for a certain period of time in the oscillation. The concentration of $\beta$-naphthalene sulfonic acid before and after adsorption in the solution is analyzed by HPLC in 1.2.The amount of adsorption is calculated according to the equation: $Q_{e}=\left(C_{0}-C_{e}\right) V / M$

\section{(Eq.1)}

$\mathrm{Q}_{\mathrm{e}}$ : the equilibrium exchange adsorption capacity $(\mathrm{mg} / \mathrm{g}), C_{0}$ : solution of the initial mass concentration $(\mathrm{mg} / \mathrm{L}), \mathrm{C}_{\mathrm{e}}$ : the concentration of solution exchange adsorption equilibrium $(\mathrm{mg} / \mathrm{L}), \quad \mathrm{V}$ : the volume of solution $(\mathrm{L}), \mathrm{M}$ : the weight of resin $(\mathrm{g})$.

\section{Results and discussion}

\section{Effect of temperature on the adsorption of resin composite}

At respectively $5^{\circ} \mathrm{C}, 10^{\circ} \mathrm{C}, 15^{\circ} \mathrm{C}, 20^{\circ} \mathrm{C}, 25^{\circ} \mathrm{C}, 30^{\circ} \mathrm{C}, 35^{\circ} \mathrm{C}$, each iodine flask with $0.1 \mathrm{~g}$ pretreatment of composite resin were added $50 \mathrm{~mL}$ of $\beta$ - naphthalenesulfonic acid solution which was $0.5 \mathrm{~g}$ $/ \mathrm{L}$, then they were oscillated in the cryostat oscillator by $150 \mathrm{rev} / \mathrm{min}$. After 24 hours the solution in each iodine flask was taken out and measured by HPLC. The equilibrium exchange adsorption is calculated by the equation (Eq.1). The adsorption capacity of different temperature as shown in figure 1. As can be read from the figure 1: with the change of temperature, the solution concentration is changed, the best exchange adsorption is not at $5^{\circ} \mathrm{C}$ but at $10^{\circ} \mathrm{C}$. The exchange adsorption amount is reduced with the increase of temperature from $10^{\circ} \mathrm{C}$ to $30^{\circ} \mathrm{C}$, which shows that the low-temperature adsorption is beneficial, but the low temperature (under $5^{\circ} \mathrm{C}$ ) affected adsorption rate. The temperature increase from $10^{\circ} \mathrm{C}$ to $25^{\circ} \mathrm{C}$, the amount of exchange adsorption is decreased, but after $30{ }^{\circ} \mathrm{C}$ the exchange capacity is increased. Increased water temperature can make the ion kinetic energy increase and the viscosity of water decrease, and can accelerate the internal diffusion and film diffusion. From the figure 1, it can be seen that the impact on the equilibrium concentration of the solution is not obvious from $15^{\circ} \mathrm{C}$ to $40^{\circ} \mathrm{C}$; below $15^{\circ} \mathrm{C}$ the work may require be cooled, so that the energy consumption is increased, so the ion exchange equipment operation need generally keep the water temperature at $20-40^{\circ} \mathrm{C}$. adsorption capacity of Hydrous ferric oxide composite anion resin can reach to $235 \mathrm{mg} / \mathrm{g}$ to $\beta$-naphthalene sulfonic acid at $10^{\circ} \mathrm{C}$.

\section{Different initial solution mass concentration effects on exchange adsorption quantity}

A series concentration of $\beta$ - naphthalene sulfonic acid solution $(100,200,300,400,500 \mathrm{mg} / \mathrm{L})$ were accurate preparated, and then added to the five $250 \mathrm{ml}$ iodine bottles respectively, $50 \mathrm{~mL}$ of $\beta$ naphthalene sulfonic acid solution() and $0.1 \mathrm{~g}$ of the dry composite resin were added to the five bottles respectively. Then the solution was oscillated more than 24 hours in the thermostatic oscillator of $150 \mathrm{r} / \mathrm{min}$ speed. When the adsorption equilibrium respectively at $10^{\circ} \mathrm{C}, 20^{\circ} \mathrm{C}, 30^{\circ} \mathrm{C}$, were ended, the concentration of $\beta$-naphthalene sulfonic acid solution was detected by HPLC. Then the influence of the initial concentration of $\beta$-naphthalene sulfonic acid solution to exchange adsorption was investigated and adsorption isotherms were plotted in figure 2 . The figure shows that 
the adsorption of exchange increases with its initial concentration increases, and shows a good linear relationship, which of the correlation coefficient was 0.99979. The increase of the initial concentration can increase the exchange adsorption equilibrium quantity. This result shows that the molecular activity of high concentration increases the chances of collision, thereby the exchange adsorption increased. Diffusion processes is relying on the ion concentration gradient driver, diffusion speed and diffusion across the interface ion concentration proportional to the difference, on both sides of the ion diffusion velocity is directly proportional to the concentration difference of diffusion interface. So the concentration of ions in the aqueous solution is an important factor affecting on diffusion.

\section{The influence of sodium chloride on the composite resin exchange adsorption}

$0 \mathrm{~mol}, 0.2 \mathrm{~mol}, 0.4 \mathrm{~mol}, 0.5 \mathrm{~mol}, 0.6 \mathrm{~mol}, 0.8 \mathrm{~mol}$ of sodium chloride was respectively added into 6 iodine bottles which were added $0.1 \mathrm{~g}$ of dry resin pretreated and then $50 \mathrm{ml} \beta$ - naphthalenesulfonic acid solution $(0.5 \mathrm{~g} / \mathrm{l})$ was added into above bottles respectively. Then at $30^{\circ} \mathrm{Cthe}$ solution was cyclotron oscillated for 24 hours in the constant temperature water bath oscillator, $150 \mathrm{rev} / \mathrm{min}$. Then the solution concentration was measured by HPLC, the effect of the results shown in Figure 3. As can be seen from the figure, with the concentration of sodium chloride was increased, the concentration of the solution of $\beta$ - naphthalenesulfonic acid also increased, but the exchange resin adsorption decreased. According to the literature [5-7], $\mathrm{NaCl}$ dissociated into positively charged $\mathrm{Na}+$ and Cl- negatively charged in water, the electric field which present around of positive and negative ions lead to there is a strong electrostatic interaction between ions and water molecules. In ternary solution of salt - solute - water, static electricity is the main reason for the presence of salt effect. if the water molecules gather around the ion but solute molecules is distant regions, the relative concentration of solute will rise, which occurred salting (salt-out), and was good for adsorption, otherwise salt dissolved (salt-in) is not conducive to absorption. For the existence of ion exchange adsorption system, anions $(\mathrm{Cl}-)$ by salt electrolysis has great influence on it. Since the formation of the competition between $\mathrm{Cl}$ - and solute, degree of influence depends on the size of the ion-exchange selectivity. If the adsorption selectivity of the resin for the solute is far higher than that of $\mathrm{Cl}-$, the impact is not obvious; If the selectivity is quite to $\mathrm{Cl}^{-}$, even lower than $\mathrm{Cl}^{-}$, the adsorption selectivity impact of the resin for the solute is adverse. Meanwhile, the factors that determine selective ion exchange include: ion valence and ionic radius. The ion valence number is higher, the electrostatic attraction of the resin functional groups is greater; the ionic radius is larger, the ion surface charge density is relatively smaller, hydrated ion radius is relatively smaller, so absorption is favorable [8].The $\beta$-naphthalene sulfonic hydrated ionic radius is relatively large, therefore the salt effect is unfavorable to exchange adsorption.

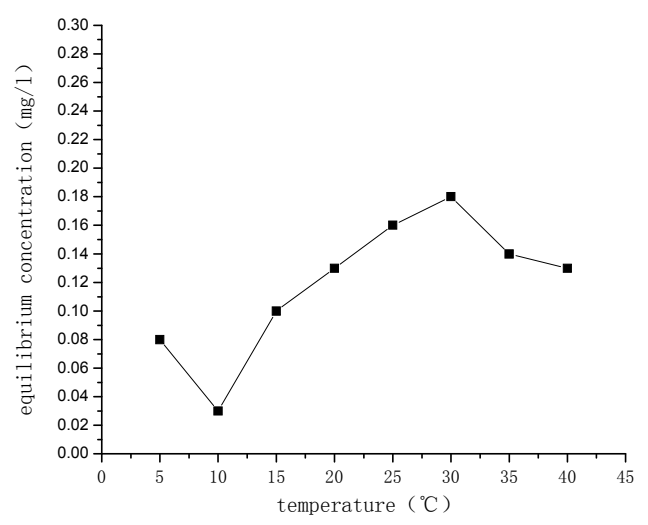

Fig.1 Effect of temperature

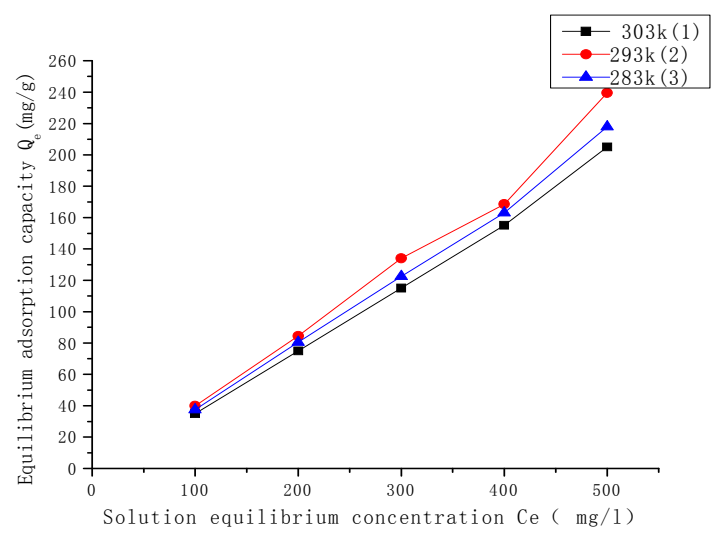

Fig.2 isothermal sorption curve 


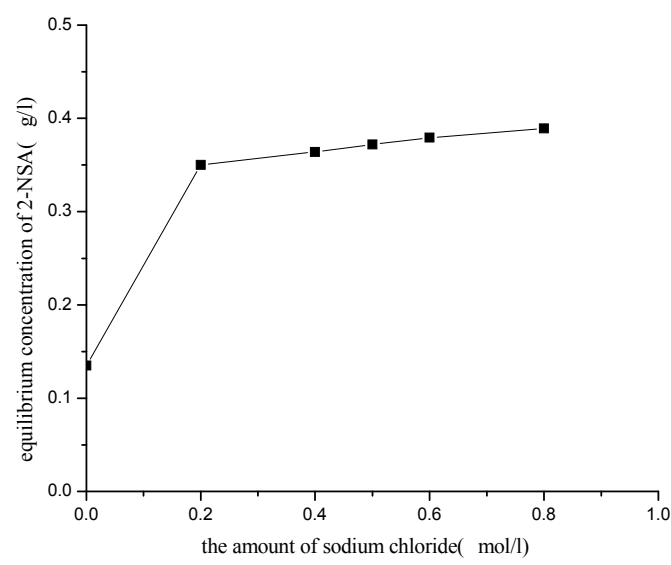

Fig.3 The influence of sodium chloride

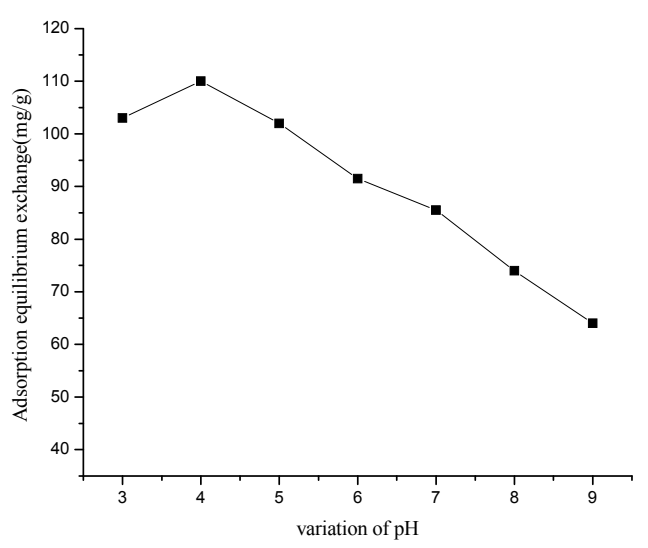

Fig.4 The influence of $\mathrm{pH}$

The influence of sodium sulfate on the composite resin exchange adsorption is shown: with the concentration of sodium sulfate was increased, the concentration of the solution of $\beta$ - naphthalene sulfonic acid increases, but the exchange resin adsorption increases only with the concentration of sodium sulfate decreases.

\section{The influence of $\mathbf{p H}$ value of composite resin exchange adsorption}

$50 \mathrm{ml} \beta$-naphthalene sulfonic acid solution $(0.5 \mathrm{~g} / \mathrm{l})$ was divided into seven iodine bottles which were added $0.1 \mathrm{~g}$ of dry resin pretreated and each were successively adjusted to $\mathrm{pH} 3,4,5,6,7,8,9$ with 5 mol / 1 sodium hydroxide solution. Then in the constant temperature water bath oscillator, a temperature of 30 degrees, $150 \mathrm{rev} / \mathrm{min}$, and shaken for 24 hours after removing the roundabout.. The solution concentration was measured by HPLC. The effect of the results shown in Figure 4 . From the figure 4 the result can be observed: at $\mathrm{pH}=4$ exchange adsorption capacity was the maximum, and with increase of $\mathrm{pH}$ value, exchange adsorption capacity was decreased; when below $\mathrm{pH}=4$, the exchange adsorption capacity was slightly lower. In the $\mathrm{pH}=4$, hydrated ferric oxide activity is the best. When the $\mathrm{pH}$ value is in the range of 4 to 6 , the surface of the iron oxide has positively charged, and the organic compounds has negatively charged in the water, so the adsorption effect is best. There is an optimized to $\mathrm{pH}$ value when the iron oxide removing organic compounds in water. When $\mathrm{pH}$ is higher, there is not enough $\mathrm{H}+$ to proton weak base groups on the surface of the resin, so the adsorption capacity is low. With the decrease in $\mathrm{pH}$, the degree of protonation of functional groups increase, so electrostatic adsorption is beneficial, on the other hand, the dissociation of the solute restrained also increased mainly in molecular form. Above reasons lead to that hydrophobic interaction was enhance, the adsorption amount was increased on the resin adsorbate [9]; When the $\mathrm{pH}$ is low to a certain extent, the $\mathrm{pH}$ of the solution reduce the adsorption of naphthalene sulfonic acid on the resin. The reason may be that low value of $\mathrm{pH}$ caused that the surface of the weak base resin has fully protonated. At the same time, the competitive adsorption of the excessive $\mathrm{SO}_{4}{ }^{2-}$ and sulfonate ions is serious[10], so the amount of adsorption reduced.

\section{Conclusion}

(1) The salt (sodium chloride or sodium sulfate) concentration of solution inhibit the adsorption of iron composite resin to the $\beta$-naphthalene sulfonic acid. With the increase of salt concentration, the adsorption capacity is decreased.

(2) The $\mathrm{pH}$ of the solution have an impact on the exchange adsorption ability of iron composite resin. HFO - D301R for $\beta$-naphthalene sulfonic acid adsorption quantity reduced with the increase of $\mathrm{pH}$

(3) The $\beta$ - naphthalene sulfonic acid aqueous solution by using the hydrated iron load weakly basic anion resin HFO-D301R and the static adsorption experiment was studied for the adsorption performance of the materials. The results showed that: HFO-D301R maximum adsorption capacity $\beta$ naphthalene sulfonic acid can reach $235 \mathrm{mg} / \mathrm{g}$ at $10^{\circ} \mathrm{C}$. 


\section{Reference}

[1] Li Changhai, Shi Pengfeil. Chemical Engineering.2001,29(6):42 -45.

[2] Shailesh M D, Sachin S P. Sep.Sci.Technol.1997, 32(7):1273 -1294.

[3] Huh,J K, Song D I. Ind. Eng. Chem. Res ., 2000, 35(2):243 -259.

[4] Li Fenggang, Ju Caixia, Li Changhai. Applied Chemical industry, 2011, 40(3):422-424.(in chinese)

[5] Huang Zhiqing, Introduction to the theory of electrolyte solution [M], Beijing: Science press, 1983.

[6] Ding Burgess, the metal ions in solution [M], Beijing: Atomic press, 1987. (in chinese)

[7] Chen Yiliang. Resin adsorption of benzene methanol production wastewater treatment of basic research and technological development [D]. Nanjing: Nanjing university Master Thesis, 2004. (in chinese)

[8] He Binglin, Hang Wenqiang. ion exchange and adsorbent resin[M].Shanghai: Shanghai science education press.1995. (in chinese)

[9] Long Chao, Zhang Quanxing, Chen Jinlong. Chinese journal of Applied chemistry [J],2004,21(10): 997-1002. (in chinese)

[10] Pan Bingcai. The adsorption properties of macroporous resin for aromatic acid and its application in 2- Naphthol in wastewater treatment [D]. Nanjing: Nanjing university doctoral dissertation 2003. (in chinese) 